\title{
Duplications of KIAA1549 and BRAF screening by Droplet Digital PCR from formalin-fixed paraffin-embedded DNA is an accurate alternative for KIAA1549-BRAF fusion detection in pilocytic astrocytomas
}

\author{
Romain Appay ${ }^{1,2} \cdot$ Frédéric Fina ${ }^{1,3} \cdot$ Nicolas Macagno ${ }^{1} \cdot$ Laëtitia Padovani $^{4} \cdot$ Carole Colin $^{2} \cdot$ Doriane Barets $^{1}$. \\ Joanna Ordioni ${ }^{1} \cdot$ Didier Scavarda $^{5}$ - Felice Giangaspero ${ }^{6,7}$ - Manuela Badiali ${ }^{8}$ - Andrey Korshunov ${ }^{9}$. \\ Stefan M. Pfister ${ }^{10,11,12} \cdot$ David T.W. Jones ${ }^{10,12}$ - Dominique Figarella-Branger ${ }^{1,2}$
}

Received: 8 December 2017 / Revised: 14 February 2018 / Accepted: 15 February 2018 / Published online: 25 May 2018

(c) United States \& Canadian Academy of Pathology 2018

\begin{abstract}
Pilocytic astrocytomas represent the most common glioma subtype in young patients and account for $5.4 \%$ of all gliomas. They are characterized by alterations in the RAS-MAP kinase pathway, the most frequent being a tandem duplication on chromosome $7 \mathrm{q} 34$ involving the $B R A F$ gene, resulting in oncogenic BRAF fusion proteins. BRAF fusion involving the KIAA1549 gene is a hallmark of pilocytic astrocytoma, but it has also been recorded in rare cases of gangliogliomas, $1 \mathrm{p} / 19 \mathrm{q}$ co-deleted oligodendroglial tumors, and it is also a common feature of disseminated oligodendroglial-like leptomeningeal neoplasm. In some difficult cases, evidence for KIAA1549-BRAF fusion is of utmost importance for the diagnosis. Moreover, because the KIAA1549-BRAF fusion constitutively activates the MAP kinase pathway, it represents a target for drugs such as MEK inhibitors, and therefore, the detection of this genetic abnormality is highly relevant in the context of clinical trials applying such new approaches. In the present study, we aimed to use the high sensitivity of Droplet Digital PCR (DDPCR ${ }^{\mathrm{TM}}$ ) to predict KIAA1549-BRAF fusion on very small amounts of formalin-fixed paraffin-embedded tissue in routine practice. Therefore, we analyzed a training cohort of 55 pilocytic astrocytomas in which the KIAA1549-BRAF fusion status was known by RNA sequencing used as our gold standard technique. Then, we analyzed a prospective cohort of 40 pilocytic astrocytomas, 27 neuroepithelial tumors remaining difficult to classify (pilocytic astrocytoma versus ganglioglioma or diffuse glioma), 15 dysembryoplastic neuroepithelial tumors, and 18 gangliogliomas. We could demonstrate the usefulness and high accuracy (100\% sensitivity and specificity when compared to RNA sequencing) of DDPCR ${ }^{\mathrm{TM}}$ to assess the KIAA1549-BRAF fusion from very low amounts of DNA isolated from formalin-fixed paraffin-embedded specimens. BRAF duplication is both necessary and sufficient to predict this fusion in most cases and we propose that this single analysis could be used in routine practice to save time, money, and precious tissue.
\end{abstract}

\section{Introduction}

Pilocytic astrocytomas account for 5.4\% of all gliomas [1]. They represent the most common glioma subtype in young patients and mainly occur within the cerebellum, although

These authors contributed equally: Romain Appay, Frédéric Fina.

Dominique Figarella-Branger

dominique.figarella-branger@univ-amu.fr

Extended author information available on the last page of the article other common locations are optic nerve, optic chiasm/hypothalamus, thalamus and basal ganglia, brain stem, and cerebral hemispheres. According to the 2016 WHO classification of central nervous system tumors, they are now classified among the group of "other astrocytic tumors" in order to emphasize that they are circumscribed in contrast to other diffuse astrocytic and oligodendroglial tumors [2, 3]. Pilocytic astrocytomas are characterized by alterations in the RAS-MAP kinase pathway, the most frequent being a tandem duplication on chromosome $7 \mathrm{q} 34$ involving the $B R A F$ gene, resulting in oncogenic $B R A F$ fusion proteins [4]. The most common partner is the KIAA1549 gene. In this case, a fragment of approximately $2 \mathrm{Mb}$ involving parts of both genes is 
duplicated and inserted at the breakpoint such that the $5^{\prime}$-end of the KIAA1549 gene becomes fused with the $3^{\prime}$-end of the $B R A F$ gene (Fig. 1). To date, nine different combinations of KIAA1549 and BRAF gene fusion have been described in the literature. The most common is a fusion between exon 16 of KIAA1549 and exon 9 of BRAF (KIAA1549-BRAF 16;9 fusion, $78 \%$ ). Less frequent variants include fusion between KIAA1549 exon 15 and BRAF exon 9 (KIAA1549-BRAF 15;9 fusion, $13 \%$ ) and KIAA1549 exon 16 and $B R A F$ exon 11 (KIAA1549-BRAF 16;11 fusion, 7\%) [5].

Because the gene fusions involve different combinations of KIAA1549 and BRAF exons, it remains difficult to identify them by RT-PCR especially from formalin-fixed paraffinembedded tissues. Moreover, because in some cases material remains scarce, technical failure is common. Recently, a study has reported the usefulness of NanoString nCounter system to detect KIAA1549-BRAF fusion from RNA extracted from formalin-fixed paraffin-embedded tissue but the amount of material needed remains high [6]. FISH analysis is widely used by some centers: it is sensitive to detect BRAF duplication but it may be difficult to interpret, especially in cases showing aneuploidy. FISH may also allow detecting the KIAA1549-BRAF fusion by using appropriate probes but here again the interpretation requires an experimented pathologist or cytogeneticist and it remains difficult in some cases. However, in some instances, evidence for KIAA1549-BRAF fusion is of utmost importance for the diagnosis.

In addition to KIAA1549-BRAF fusion status, other genetic alterations might be of interest to be searched for in DNA extracted from low-grade gliomas such as FGFRl or $B R A F$ point mutation or $F G F R l$ duplication as examples. Therefore, a technique that would allow KIAA1549-BRAF fusion detection on DNA extracted from small amount of formalin-fixed paraffin-embedded samples would be of substantial value in routine practice. DDPCR ${ }^{\mathrm{TM}}$ represents a powerful single-molecule counting strategy to detect minute amounts of genetic material with performance surpassing many quantitative methods. It allows the measuring of low amplitude Copy Number Variation and the accurate counting of alleles from DNA isolated from a mixture of heterogeneous cell populations and from highly degraded DNA prepared from formalin-fixed paraffin-embedded tissue samples [7]. This technique was successfully used in our department for the detection of several molecular alterations in different diseases including the detection of the internal tandem duplication of the tyrosine-kinase domain of FGFRl gene in dysembryoplastic neuroepithelial tumors [8]. In the present study, we wanted to utilize the sensitivity of DDPCR ${ }^{\mathrm{TM}}$ to show the occurrence of gene duplications on very small amounts of formalin-fixed paraffin-embedded tissue in routine practice. Because the KIAA1549-BRAF gene fusion is the consequence of a tandem duplication involving parts of both genes, we hypothesized that the identification of duplicated regions in these two genes was directly correlated to the detection of fusion transcripts. Moreover, the duplication of KIAA1549 exon 16 suggest the occurrence of a KIAA1549-BRAF fusion transcript involving exon 16 (Fig. 1).

To test this hypothesis, we first used a training cohort of 55 pilocytic astrocytomas in which the KIAA1549-BRAF fusion status was known (RNA sequencing on frozen specimen). In this cohort of patients we performed DDPCR ${ }^{\mathrm{TM}}$ from DNA samples extracted from formalin-fixed paraffinembedded tissues. The sensitivity and specificity of this method for each designed assay were compared against gold standard RNA-sequencing analysis. In addition, we analyzed the KIAA15149-BRAF fusion status in a prospective cohort of 40 pilocytic astrocytomas, 27 low-grade neuroepithelial tumors remaining difficult to classify (pilocytic astrocytoma versus ganglioglioma and pilocytic astrocytoma versus diffuse glioma), 15 dysembryoplastic neuroepithelial tumors, and 18 gangliogliomas.

\section{Materials and methods}

\section{Patients}

\section{Training cohort}

The first series involved formalin-fixed paraffin-embedded DNA samples from 31 pilocytic astrocytomas with available KIAA1549-BRAF fusion status (RNA-sequencing analysis on frozen specimen) provided by the German Cancer Research Center in Heidelberg. Among them, 16 cases were KIAA1549-BRAF fused and 15 were not fused. Regarding these 15 cases, 2 of them displayed trisomy 7. The second series, also provided by the German Cancer Research Center included formalin-fixed paraffin-embedded scrolls from 19 pilocytic astrocytoma samples, all displaying KIAA1549$B R A F$ fusion status demonstrated by RNAseq (RNAsequencing analysis performed on frozen specimen).

We also analyzed DNA from five additional gliomas demonstrating partial chromosome $7 \mathrm{q}$ gain. Therefore, the training cohort comprised a total of 55 cases: 20 not-fused cases (2 trisomy 7 and 5 7q gain), 9 KIAA1549-BRAF 15;9 fusion cases, 26 cases involving KIAA1549 exon 16 fusion (3 with 16;11 fusion and 23 with 16;9 fusion).

\section{Prospective cohort}

The second cohort comprised samples from 100 French patients diagnosed as pilocytic astrocytomas (40 cases), gangliogliomas (18 cases), dysembryoplastic neuroepithelial tumors (15 cases), and 27 neuroepithelial tumors that remained difficult to classify (gangliogliomas versus pilocytic 


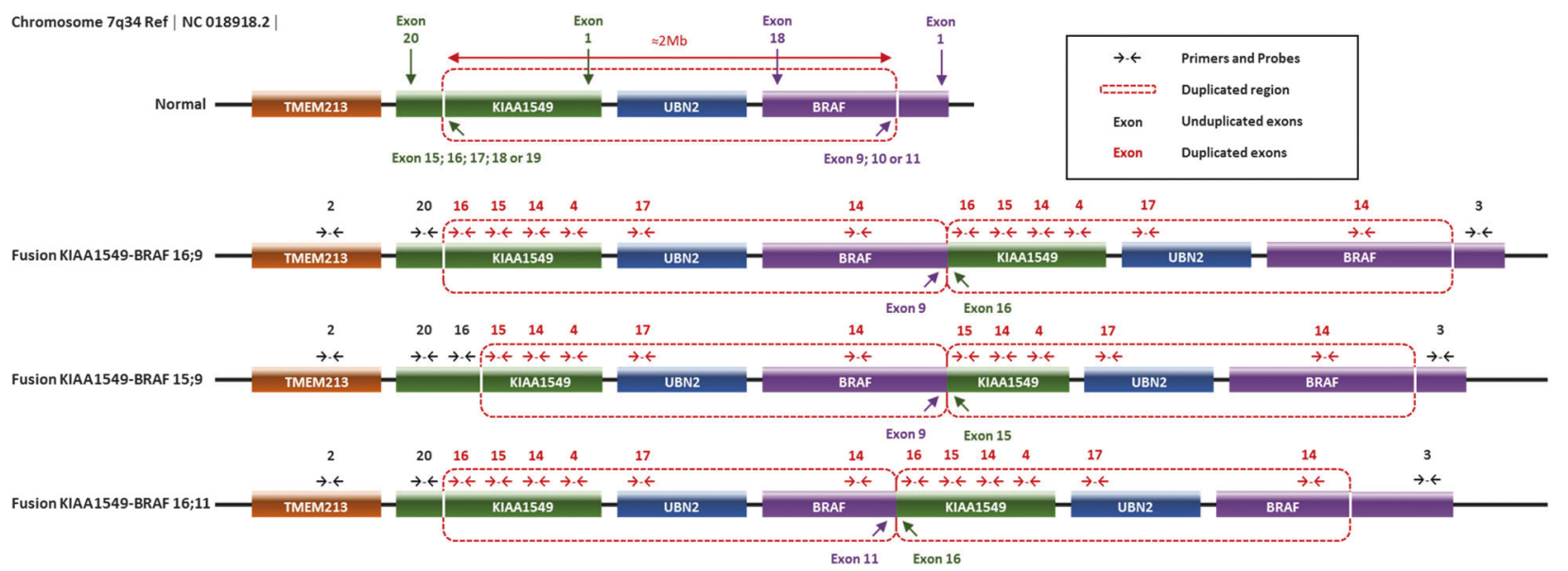

Fig. 1 Schematic representation of the normal portion of chromosome 7q34 including KIAA1549, UBN2, and BRAF genes and the gene fusion event including some exons of BRAF and KIAA1549 genes.
UBN2 gene has been described to be affected by this duplication whereas TMEM213 gene is not

was above $60 \%$. Then, tumor DNA was extracted from $4 \times 5$ $\mu \mathrm{m}$ thick sections of formalin-fixed paraffin-embedded tissue samples after dewaxing, as previously described [9]. The diameter of the formalin-fixed paraffin-embedded sections varied from $5 \mathrm{~mm}$ to $2 \mathrm{~cm}$.

\section{RNA sequencing}

RNA sequencing was performed on frozen tissue in Heidelberg (German Cancer Research Center) as previously described [10].

\section{KIAA1549-BRAF fusion status}

In order to search for a duplication of the KIAA1549 and $B R A F$ genes that would be associated with the KIAA1549$B R A F$ fusion we developed droplet digital PCR (DDPCR ${ }^{\mathrm{TM}}$, Bio-rad) assays to screen copy number variation duplications. Therefore, we designed our assay to quantify DNA copy number of KIAA1549 (exons 4, 14, 15, and 16) and $B R A F$ (exon 14) and compared the results respectively with DNA copy number of KIAA1549 (exon 20) and BRAF (exon 3) that are described to be not affected by the duplication (Fig. 1) [11]. Moreover, because the duplication, if it exists, also affects the Ubinuclein 2 (UBN2) gene but not the Transmembrane protein 213 (TMEM213) gene (Fig. 1), we also designed probes targeting UBN2 exon 17 and TMEM213 intron 2 and we compared DNA copy number of $U B N 2$ exon 17 with DNA copy number of TMEM213 intron 2 in order to confirm the duplication. As previously reported [8], we theoretically considered a sample as duplicated if the mean copy number variation value of the ratio between the two exons analyzed was above 2.25 and the copy number variation min above 2.00 (CI 95\%, Poisson law), with a minimum DNA 

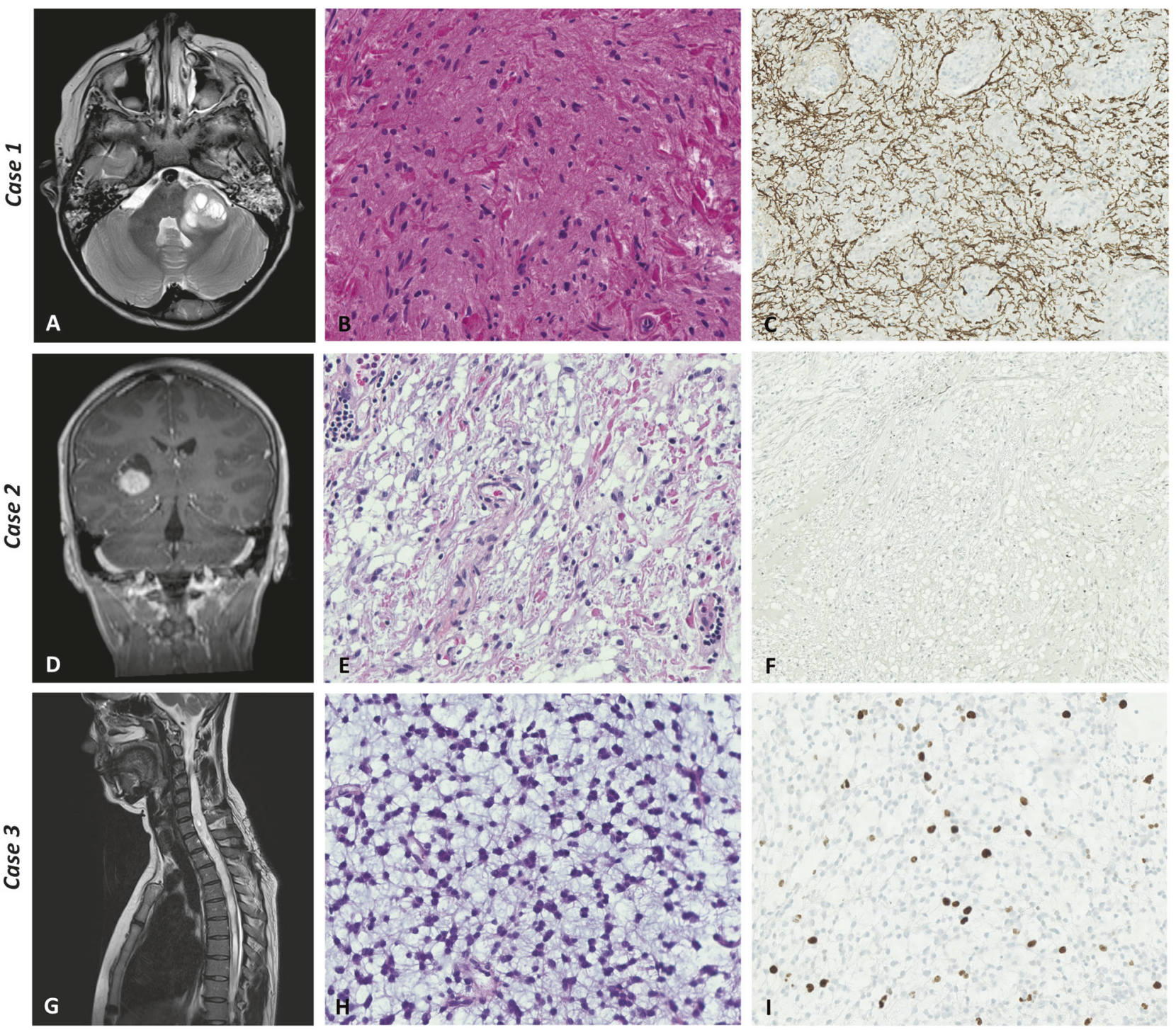

Fig. 2 Example of three cases difficult to classify. Case 1: a T2 weighted magnetic resonance imaging (MRI) showing a well limited tumor located in the cerebellum. b Hematoxylin and eosin (HE) stained section demonstrating a proliferation of elongated bipolar glial cells associated with numerous Rosenthal fibers $(\mathrm{HE}, \times 40)$. c Presence of numerous axons revealed by anti-neurofilament immunostaining $(\times 20)$. In this case, KIAA1549-BRAF fusion was recorded and final diagnosis was pilocytic astrocytoma. Case 2: d Temporal cystic lesion with a mural nodule enhanced after gadolinium injection on MRI. e Proliferation of elongated cells associated with Rosenthal fibers and eosinophilic granular bodies and perivascular lymphocytic infiltrate (HE, $\times 40$ ). f Neurofilament immunostaining reveals absence of neuronal component $(\times 20)$. This case exhibit neither KIAA1549-BRAF fusion nor $B R A F \mathrm{~V} 600 \mathrm{E}$ mutation and final diagnosis remains elusive. Case 3: g T2 weighted MRI showing a well-circumscribed spinal tumor. h Proliferation of monomorphous bipolar cells within a myxoid background (HE, $\times 40$ ). i High proliferation index evaluated by antiKi67 antibody $(\times 40)$. KIAA1549-BRAF fusion was recorded and final diagnosis was pilocytic astrocytoma concentration of 100 copies $/ \mu \mathrm{L}$ of DNA $(330 \mathrm{pg} / \mu \mathrm{L})$ analyzed with 35.000 nano-droplets of DDPCR ${ }^{\mathrm{TM}}$.

DDPCR $^{\mathrm{TM}}$ analysis was performed as previously described [8]. Briefly, each 21- $\mu$ l reaction mixture contained $5 \mu \mathrm{L}$ of DNA template, $2 \times$ DDPCR $^{\mathrm{TM}}$ supermix for probes (no dUTP) and BRAF exon 14 (FAM $^{\mathrm{TM}}$ ) and exon 3 $\left(\mathrm{HEX}^{\mathrm{TM}}\right)$ assays, or KIAA1549 exon 4 (FAM $\left.{ }^{\mathrm{TM}}\right)$ and exon 20 (HEX $^{\mathrm{TM}}$ ), or KIAA1549 exon 14 (FAM ${ }^{\mathrm{TM}}$ ) and exon 20 (HEX $\left.{ }^{\mathrm{TM}}\right)$, or KIAA1549 exon 15 (FAM $\left.{ }^{\mathrm{TM}}\right)$ and exon 20
(HEX $\left.^{\mathrm{TM}}\right)$, or KIAA1549 exon $16\left(\mathrm{FAM}^{\mathrm{TM}}\right)$ and exon 20 (HEX $\left.{ }^{\mathrm{TM}}\right)$, or $U B N 2$ exon 17 (FAM $\left.^{\mathrm{TM}}\right)$ and TMEM213 intron $2\left(\mathrm{HEX}^{\mathrm{TM}}\right)$. The assays were purchased as a $20 \times$ premix of primers and probes and used at $1 \times$ concentration (Bio-Rad Laboratories). The $1 \times$ concentration of this assay comprised $900 \mathrm{nM}$ forward primer, $900 \mathrm{nM}$ reverse primer, and $250 \mathrm{nM}$ probe. Experiments were performed in triplicate for each sample. Primers, hydrolysis probe sequences and DDPCR ${ }^{\mathrm{TM}}$ conditions are reported in Table 1. 
Table 1 Primers, hydrolysis probes sequences and DDPCR ${ }^{\mathrm{TM}}$ conditions for the detection of UBN2, TMEM213, BRAF, and KIAA1549 copy number variations, reflecting duplication of these genes and at last, KIAA1549-BRAF fusion event

Primers and FAM probe sequences for exon 17 UBN2 detection

Primers and HEX probe sequences for intron 2 TMEM213 detection

Primers and HEX probe sequences for exon 3 BRAF detection

Primers and FAM probe sequences for exon 14 BRAF detection

Primers and FAM probe sequences for exon 4 KIAA1549 detection

Primers and FAM probe sequences for exon 14 KIAA1549 detection

Primers and FAM probe sequences for exon 15 KIAA1549 detection

Primers and FAM probe sequences for exon 16 KIAA1549 detection

Primers and HEX probe sequences for exon 20 KIAA1549 detection

Thermocycler conditions
Forward: 5'-GGAGCTCAGCATGCAGCAA -3'

Reverse: 5'-GATGAAGGAGGTGGCGTTCTC-3'

Probe: 5'-ACTCACCTCTGCCTGCACACTTACAGCAAG-3'

Forward: 5'-CAACAGCAAAGTGGAAGAAAGGAT-3'

Reverse: 5'-CTCCTGGTCATGTCCCAGTTC-3'

Probe: 5'-CCGCATTCTACAGGATCGGCCTTAGC-3'

Forward: 5'-CAAGTCACCACAAAAACCTATCGT-3'

Reverse: 5'-ATACAAAGAAACAGCAAAATGGTGAT-3'

Probe: 5'-AGAGTCTTCCTGCCCAACAAACAGAGGAC-3'

Forward: 5'-AAATGATGTTTTGTTTTTCAGTTACTT-3'

Reverse: 5'-GCAGACAAATTTCAGGAAGGA-3'

Probe: 5'-ACGCCAAGTCAATCATCCACAGAGA-3'

Forward: 5'-TGTCCGTGACCAGACTCCTTT-3'

Reverse: 5'-TGTCTCACCTGTTTGAACTTGGAA-3'

Probe: 5'-CCTGTCTGTGAAACCTTCTTTCCTTGTGCC-3'

Forward: 5'-CGGGACCTCTATGGCTGTCTT-3'

Reverse: 5'-GGTGCTCGATCTCAGACTTGTG-3'

Probe: 5'-TTCCTCAGATTCAGACCGCGCTGC-3'

Forward: 5'-AAGCCTCGCCGGAAACA-3'

Reverse: 5'-CATCGCTGTCTGTGGTGATGA -3'

Probe: 5'-TCAACGGCTGTCCTGCCGACG-3'

Forward: 5'-TCCTTTGCCATTTCTCATTGTAGTC-3'

Reverse: 5'-CTGGATACCTCCCCAGTTCCA-3'

Probe: 5'-CAGCCGATGTGCAGACACCATCCTC-3'

Forward: 5'-GCACCGTGCAGAACTTCCA-3'

Reverse: 5'-GTTGATTTCCTTTTGGTCTTGCTT-3'

Probe: 5'-CAGATTTGCCAAGTATCCGCTTCCTGTG-3'

$95^{\circ} \mathrm{C}, 10 \mathrm{~min}(1 \mathrm{cycle})$

$94^{\circ} \mathrm{C}, 30 \mathrm{~s}-55^{\circ} \mathrm{C}, 1 \mathrm{~min}$ (40 cycles)

$98^{\circ} \mathrm{C}, 10 \min (1$ cycle $)$

$15^{\circ} \mathrm{C} \infty$

Use of a heated lid set to $105^{\circ} \mathrm{C}$

\section{Defining limits of BRAF exon 14/exon 3 duplication detection}

In order to determine the limits of detection of this $\mathrm{DDPCR}^{\mathrm{TM}}$ assay, we empirically characterized the limits of detection of $B R A F$ exon 14/exon 3 duplication, by determining the amounts of DNA needed regarding different copy number variation values. The copy number variation represents for a haploid genome the ratio of target DNA to reference DNA multiplied by two. To achieve our goal, we made four mixtures from several pools of DNA from the training cohort with known KIAA1549:BRAF duplication status. One with a pool of samples displaying duplicated DNA BRAF exon 14/exon 3 (ratio of target DNA to reference $\mathrm{DNA}=1.385$, copy number variation $=2.77$ ). Note that a duplicated sample with one additional allelic copy would have a theoretical copy number variation of 3.0, so this pool displaying a copy number variation of 2.77 corresponds in theory to a duplication in $77 \%$ of the cells and likely reflects $<100 \%$ tumor purity as well as possibly some intra-tumoral heterogeneity of presence of the fusion. A second pool of samples without BRAF exon 14/exon 3 duplication (ratio of target DNA to reference DNA $=0.98$; copy number variation $=1.96$ ) was also generated. The last two pools were obtained by mixing DNA from the first and second DNA pools, displaying respectively copy number variation values of 2.33 and 2.21 in order to frame the cut-off value copy number variation $=2.25$. Each mixture was then diluted in cascade, to a theoretical minimum detection value of $300 \mathrm{pg}$ of total DNA per DDPCR ${ }^{\mathrm{TM}}$ assay (Fig. 3).

\section{Statistical analysis}

The $\chi^{2}$ test (or Fisher's exact test, as appropriate) was used to correlate the following variables: histological diagnosis and copy number variation results. The test was two-sided and the statistical significance was defined as $p<0.05$. Analyses were conducted using PASW Statistics version 17.02 (IBM SPSS Inc., Chicago, IL, USA). 


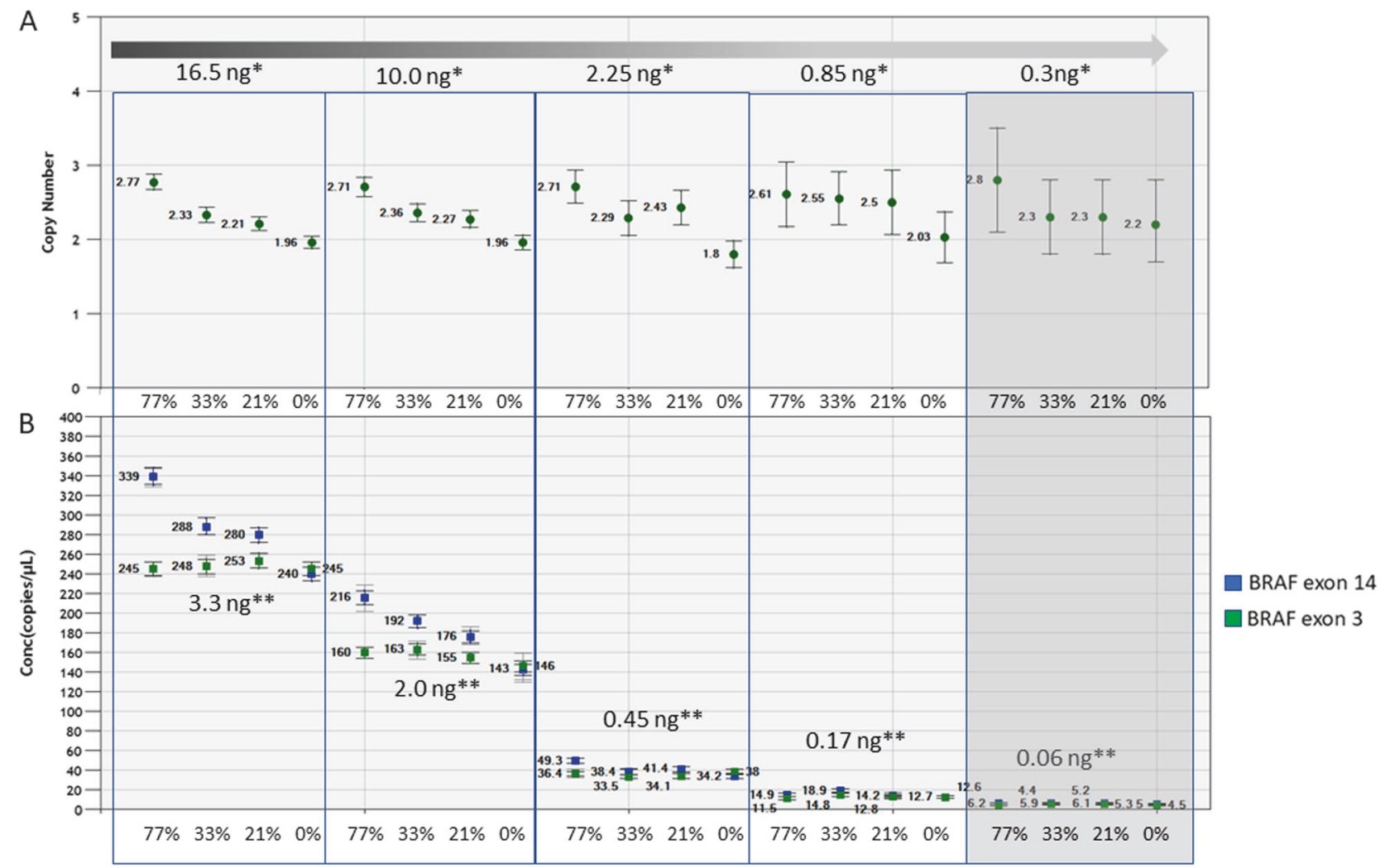

Fig. 3 Empirical characterization of the DDPCR ${ }^{\mathrm{TM}}$ limits of detection for $B R A F$ exon 14/exon 3 duplication by determining the minimal amounts of DNA needed regarding different copy number variation values, in particular around the theoretical threshold value of copy number variation $=2.5$ (copy number variation $\min 2.0$; CI:95\%, Poisson Law). The first copy number variation values (2.77) for the duplicated DNA pool corresponds to a ratio of 1385 and means in theory a monoallelic duplication in $77 \%$ of tumor cells. 33 and $21 \%$ were obtained by mixing duplicated and normal DNA pool in order to frame the cut-off value copy number variation $=2.25$ corresponding to a duplication in $25 \%$ of tumor cells; $0 \%$ corresponds to the normal
DNA pool. The gray gradient arrow corresponds to a dilution of each mixed and normal DNA at $12.5 \mathrm{ng}, 10.0 \mathrm{ng}, 2.25 \mathrm{ng}, 0.85 \mathrm{ng}$, and 0.3 ng total DNA in the assay ( $*=5 \mu \mathrm{L}$ DNA). a copy number variation values for each dilution of mixed and normal samples. b Concentrations expressed in copies/ $\mu \mathrm{L}$ of DDPCR ${ }^{\mathrm{TM}}(20 \mu \mathrm{L})$ for $B R A F$ exon 14 (blue) and $B R A F$ exon 3 (green) for each dilution of mixed and normal samples, ** corresponds to the concentration of DNA expressed in ng/ $\mu \mathrm{L}$ of DNA samples. The limit of detection is shown schematically by the gray vertical rectangle, it borders the value of $0.3 \mathrm{ng}$ of total DNA to which the values of copy number variation $\geq 2.25$; copy number variation $\min \geq 2.0$ (CI 95\% Poisson law) are no longer respected

\section{Results}

In this study we aimed to set up a robust method to search for KIAA1549-BRAF fusion status by DDPCR ${ }^{\mathrm{TM}}$ on formalin-fixed paraffin-embedded DNA samples. To achieve this goal, we first analyzed $B R A F$ duplication status, UBN2 duplication status, KIAA1549 and TMEM213 duplication status in the training cohort. We used RNA sequencing as the gold standard method to calculate the sensitivity and specificity of each DDPCR $^{\mathrm{TM}}$ assay and to define the best assay to detect KIAA1549-BRAF fusion. We also searched for $K I A A 1549-B R A F$ fusion status by DDPCR ${ }^{\mathrm{TM}}$ in the prospective cohort.

\section{Training cohort (55 cases) (Table 2 )}

\section{BRAF gene duplication status}

Copy number variation value of $B R A F$ exon 14 over $B R A F$ exon 3 was calculated for all samples. All cases exhibiting KIAA1549-BRAF fusion by RNAseq demonstrated BRAF gene duplication (35/35). However, none of the non-fused cases assessed by RNAseq displayed BRAF gene duplication (20/20). Importantly, none of the seven cases that displayed chromosome 7 trisomy (two cases) or partial chromosome 7q gain (five cases) displayed BRAF gene duplication relative to the adjacent control locus. 
Table 2 Results of the DDPCR ${ }^{\mathrm{TM}}$ assay performed on the training cohort

\begin{tabular}{|c|c|c|c|c|c|c|c|}
\hline Case & $\begin{array}{l}\text { KIAA1549- } \\
\text { BRAF fusion } \\
\text { (RNAseq) }\end{array}$ & $\begin{array}{c}\text { BRAF } \\
\text { exon } 14 \\
\text { duplication }\end{array}$ & $\begin{array}{c}\text { UBN2 } \\
\text { duplication }\end{array}$ & $\begin{array}{c}\text { KIAA1549 } \\
\text { exon } 4 \\
\text { duplication }\end{array}$ & $\begin{array}{c}\text { KIAA1549 } \\
\text { exon } 14 \\
\text { duplication }\end{array}$ & $\begin{array}{c}\text { KIAA1549 } \\
\text { exon } 15 \\
\text { duplication }\end{array}$ & $\begin{array}{c}\text { KIAA1549 } \\
\text { exon } 16 \\
\text { duplication }\end{array}$ \\
\hline 71 & 15_9 & + & + & + & + & + & - \\
\hline 98 & 15_9 & + & + & + & + & + & - \\
\hline 141 & 15_9 & + & + & + & - & + & - \\
\hline 146 & 15_9 & + & + & + & + & + & - \\
\hline 216 & 15_9 & + & + & + & - & - & - \\
\hline 283 & 15_9 & + & + & + & + & + & - \\
\hline 463 & 15_9 & + & + & + & + & + & - \\
\hline 514 & 15_9 & + & + & + & + & + & - \\
\hline 80910 & 15_9 & + & + & + & + & + & - \\
\hline 218 & 16_11 & + & + & + & + & + & + \\
\hline 312 & 16_11 & + & + & + & + & + & + \\
\hline 78640 & 16_11 & + & + & + & + & + & + \\
\hline 90 & 16_9 & + & + & + & + & + & + \\
\hline 489 & 16_9 & + & + & + & - & + & + \\
\hline 549 & 16_9 & + & + & + & - & - & - \\
\hline 551 & 16_9 & + & + & + & - & + & + \\
\hline 553 & 16_9 & + & + & + & + & + & + \\
\hline 554 & 16_9 & + & + & + & + & + & + \\
\hline 558 & 16_9 & + & + & + & - & + & + \\
\hline 582 & 16_9 & + & + & + & + & + & + \\
\hline 592 & 16_9 & + & + & + & - & + & + \\
\hline 79886 & 16_9 & + & + & + & - & + & + \\
\hline 80528 & 16_9 & + & + & + & + & + & + \\
\hline 83010 & 16_9 & + & + & - & + & + & + \\
\hline 83616 & 16_9 & + & + & + & + & + & + \\
\hline 83618 & 16_9 & + & + & + & + & + & - \\
\hline 84180 & 16_9 & + & + & + & + & + & + \\
\hline 84816 & 16_9 & + & + & + & - & + & + \\
\hline 90482 & 16_9 & + & + & + & + & + & + \\
\hline 91776 & 16_9 & + & + & + & - & + & + \\
\hline 92186 & 16_9 & + & + & + & - & + & - \\
\hline 92512 & 16_9 & + & + & + & + & + & + \\
\hline 92524 & 16_9 & + & + & + & + & + & + \\
\hline 93570 & 16_9 & + & + & + & + & + & + \\
\hline 93578 & 16_9 & + & + & + & + & + & + \\
\hline
\end{tabular}

Grey cells correspond to unexpected results regarding RNA seq gold standard 


\section{UBN2 gene duplication status}

$U N B 2$ gene duplication status was assessed by the ratio of UBN2 over TMEM213. All cases exhibiting KIAA1549$B R A F$ fusion by RNAseq demonstrated UBN2 gene duplication (35/35). Again, none of the seven cases with numerical abnormalities of chromosome 7 displayed UBN2 gene duplication.

Sensitivity and specificity of $B R A F$ exon 14 and UBN2 gene duplication status assessed by DDPCR ${ }^{\mathrm{TM}}$ as compared to RNAseq as the gold standard to detect KIAA1549-BRAF fusion were $100 \%$.

\section{KIAA1549 gene duplication status}

To investigate the KIAA1549 duplication status, we used in a first step three sets of primers, allowing us to calculate the following copy number variation ratio: KIAA1549 exon 14/ KIAA1549 exon 20, KIAA1549 exon 15/KIAA1549 exon 20, and KIAA1549 exon 16/KIAA1549 exon 20.

According to Fig. 1, we postulated that in all KIAA1549$B R A F$ fused cases, duplication of KIAA1549 exon 14/ KIAA1549 exon 20 and KIAA1549 exon $15 /$ KIAA1549 exon 20 should be present. Duplication of and KIAA1549 exon 16/KIAA1549 exon 20 might be observed only in fusion cases showing a KIAA1549-BRAF transcript involving exon 16.

1. Unexpectedly, duplication of KIAA1549 exon 14/ KIAA1549 exon 20 was only recorded in $24 / 35$ cases displaying fusion transcripts by RNAseq. Although we have no clear explanation for this technical failure it might be secondary to the primer set designed or to a genomic instability in this peculiar genomic sequence. Among the 15 cases displaying no fusion by RNAseq, we recorded one case with the KIAA1549 exon 14/KIAA1549 exon 20 duplication. Of importance, this case did not show $B R A F$ duplication. This assay compared to the RNAseq results only showed a sensitivity and a specificity of 68.6 and $93.3 \%$, respectively.

2. Duplication of KIAA1549 exon 16/KIAA1549 exon 20 was recorded in 23/35 cases. Interestingly all cases showing KIAA1549-BRAF 15;9 fusion by RNAseq did not have KIAA1549 exon 16/KIAA1549 exon 20 duplication (9/9). Surprisingly, among the 23 cases that demonstrated KIAA1549-BRAF 16;9 fusion by RNAseq, 3 cases did not show this duplication. This assay compared to RNAseq results showed a sensitivity and a specificity of 88.5 and $100 \%$, respectively.

3. Duplication of KIAA1549 exon 15/KIAA1549 exon 20 was recorded in $31 / 35$ cases. One of the negative cases demonstrated KIAA1549-BRAF 15;9 fusion by RNAseq, and the three others a KIAA1549-BRAF 16;9 fusion. This assay compared to RNAseq results showed a sensitivity and a specificity of 88.6 and $100 \%$, respectively.

4. We hypothesized that the lack of sensitivity of these three assays might be attributed to the variability of the involved region since the targeted exons are located near to the breakpoint junction. Because KIAA1549 exon 4 is closer to UBN2 than exon 14 and because in all cases described in the literature, exon 4 is always fused, we then decided to analyze and calculate the KIAA1549 exon 4/KIAA1549 exon 20 copy number variation ratio. Only one case did not show duplication but it demonstrated a mean copy number variation value of the ratio of 2.23 , very close to the cut-off value of 2.25. All other cases (34/35) exhibited this duplication, whereas the 15 cases displaying no fusion by RNAseq did not (15/15). This assay, compared to the RNAseq gold standard reached a sensitivity of $97.1 \%$ with always a specificity of $100 \%$.

\section{Definition of KIAA1549-BRAF fusion status according to DDPCR $^{\text {TM }}$}

In summary, for this cohort of 55 samples, none of the 20 non-fused cases showed BRAF exon 14 and KIAA1549 exon 4 duplication, and only one case was recorded with KIAA1549 exon 14 duplication but normal exon 4 (although the values for the latter were very close to the threshold for scoring duplication). All RNAseq-fused cases (35) were $B R A F$ exon 14 duplicated and $34 / 35$ were KIAA1549 exon 4 duplicated. Of note, this discrepant case (83010) was KIAA1549 exon 14, 15, and 16 duplicated. Since all KIAA1549-BRAF fused cases demonstrated BRAF (and $U B N 2$ ) duplication, we could state that if a sample did not demonstrate $B R A F$ duplication, KIAA1549-BRAF fusion did not occur.

\section{Defining limits of BRAF exon 14/exon 3 duplication detection}

In order to determine the limits of detection of this $\mathrm{DDPCR}^{\mathrm{TM}}$ assay, we have empirically characterized the minimal amounts of DNA needed to detect the $B R A F$ exon 14/exon 3 duplication according to different copy number variation values. The results showed that one nanogram of amplifiable DNA is sufficient to detect the amplification if this alteration is present in at least $25 \%$ of the total amounts of analyzed cells (this corresponds to the cut-off copy number variation value $=2.25)$. Although the standard error 


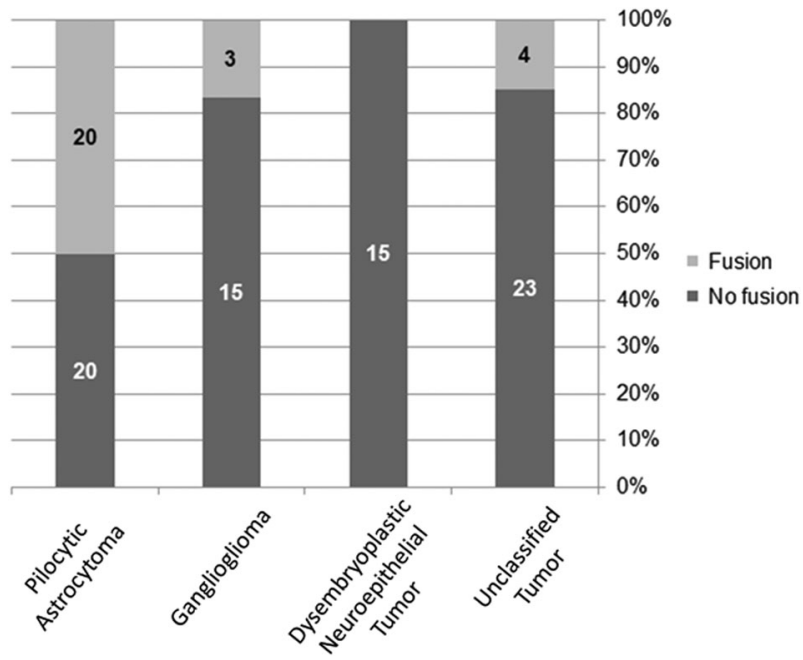

Fig. 4 Correlation between KIAA1549-BRAF fusion detection by $\mathrm{DDPCR}^{\mathrm{TM}}$ and tumor type in the prospective cohort $(n=100)$

increases when the total amounts of DNA decrease it still gives significant results with $1 \mathrm{ng}$ of DNA (Fig. 3).

\section{Prospective cohort (100 cases)}

When we started to study the prospective cohort, we analyzed by DDPCR ${ }^{\mathrm{TM}} B R A F$ exon 14 over $B R A F$ exon 3 and KIAA1549 exon 14 over KIAA1549 exon 20.

Duplication data were not exploitable for five cases; this represents a relatively low failure rate $(5 \%)$ given the difficulties commonly encountered with formalin-fixed paraffin-embedded sample analysis. BRAF duplication was recorded in $20 / 40$ pilocytic astrocytomas (50\%), 3/18 gangliogliomas (16.7\%), 0/15 dysembryoplastic neuroepithelial tumors, and in 4/27 tumors difficult to classify (Fig. 4): for one of them, case 1 (Fig. 2a-c), the diagnosis could not be made between pilocytic astrocytoma and ganglioglioma, and for the three other cases, although the pathological features were compatible with a pilocytic astrocytoma, a diagnosis of infiltrating glioma could not be ruled out as illustrated by case 3 (Fig. $2 \mathrm{~g}-\mathrm{i}$ ). In some cases difficult to classify (pilocytic astrocytoma versus ganglioglioma), KIAA1549-BRAF fusion was not recorded and diagnosis remains elusive (case 2, Fig. 2d-f). According to the results of the training cohort, we could extrapolate that the 27 cases exhibiting $B R A F$ duplication displayed KIAA1549-BRAF fusion. In accordance, 25/27 cases also displayed a KIAA1549 exon 14/ KIAA1549 exon 20 duplication. Importantly, the two negative cases displayed a duplication of KIAA1549 exon 4/KIAA1549 exon 20. Therefore, in accordance with the literature, KIAA1549$B R A F$ fusion detection was significantly linked to the pilocytic astrocytoma diagnosis $(p=0.004)$. Of note, we observed a KIAA1549 exon 14/KIAA1549 exon 20 duplication in 18 cases that did not demonstrate $B R A F$ duplication. It was not possible to test KIAA1549 exon 4/KIAA1549 exon 20 duplication in these cases because DNA as well as formalin-fixed paraffin-embedded material were no longer available. The histological diagnoses of these tumors were: six pilocytic astrocytomas, seven gangliogliomas, four dysembryoplastic neuroepithelial tumors, and one unclassified glioma. According to the criteria defined above, these cases would be considered as fusion negative.

\section{Discussion}

In this study, we report the usefulness of DDPCR ${ }^{\mathrm{TM}}$ to predict KIAA1549-BRAF fusion from very low amounts of DNA isolated from formalin-fixed paraffin-embedded specimens. In our reference cohort, this method displayed $100 \%$ sensitivity and specificity when compared to RNA sequencing as a "gold standard". Because DDPCR TM is highly sensitive, we were able to assess the KIAA1549$B R A F$ fusion in a sample with a minimum of $850 \mathrm{pg}$ of total DNA per DDPCR ${ }^{\mathrm{TM}}$ assay. We also showed that BRAF duplication was both necessary and sufficient to predict KIAA1549-BRAF fusion in most cases and we proposed that this single analysis could be used in routine practice to save time, money and precious tissue. In keeping with this result, many centers accept the demonstration of a duplication at $7 \mathrm{q} 34$ encompassing the $B R A F$ gene (usually by FISH) as evidence of the KIAA1549-BRAF fusion. Importantly, other less frequent fusions involving $B R A F$ gene have been reported in pilocytic astrocytomas such as FAM131-BRAF, RNF130-BRAF, CLCN6-BRAF, MKRN1-BRAF, GNAI1$B R A F$, and more recently GTF2I-BRAF [10, 12-14]. GTF2I-BRAF and MKRN1-BRAF fusions on one hand, and FAM131-BRAF fusion on the other hand are formed respectively by tandem duplication and interstitial deletion on chromosome arm 7q34 (involving BRAF). In these extremely rare cases, DDPCR ${ }^{\mathrm{TM}}$ might show a $B R A F$ duplication or deletion without KIAA1549 duplication, whereas the other fusions are the result of translocations or inversions and thus are not associated with copy number variation of the BRAF gene. KIAA1549-BRAF fusion is a hallmark of pilocytic astrocytomas [4].

As shown in Table 3, the use of the DDPCR ${ }^{\mathrm{TM}}$ to demonstrate the KIAA1549-BRAF fusion offers several advantages over the various commonly used techniques. This system is of interest in routine practice since results can be obtained quickly and with a high success rate even on small and damaged formalin-fixed paraffin-embedded samples. In addition, it allows searching for other molecular anomalies of interest from the extracted DNA. The sensitivity and specificity of the DDPCR ${ }^{\mathrm{TM}}$ is high in comparison with RNAseq, nanostring, and CGH-array but it allows the evaluation of only a limited number of genetic abnormalities. As compared to the DDPCR ${ }^{\mathrm{TM}}$, FISH presents the main advantage of not requiring any expensive equipment, but its performances are mainly dependent on the operator. 
Although not all pilocytic astrocytomas display the KIAA1549-BRAF fusion, it is extremely common in the cerebellum (up to $90 \%$ of cases for some authors [4]), but less frequent in supratentorial locations. KIAA1549-BRAF fusion has also been recorded in rare cases of gangliogliomas [15], 1p/19q co-deleted oligodendrogliomas [16], and it is also a common feature of diffuse leptomeningeal glioneuronal tumors (DLGNT [17],). Interestingly these three diagnoses represent differential diagnoses for pilocytic astrocytoma. In fact, pathological diagnosis of pilocytic astrocytoma is easy when it demonstrates typical biphasic architecture and Rosenthal fibers but it might be tricky in cases exhibiting monomorphous appearance of cells closely resembling oligodendrocytes. When such a pattern is obvious, differential diagnosis might be discussed regarding the location of the tumors. When the tumor demonstrates a typical leptomeningeal growth, diagnosis of DLGNT must be discussed. These cases usually demonstrate $1 \mathrm{p}$ deletion or $1 \mathrm{p} / 19 \mathrm{q}$ codeletion (but no $I D H$ gene mutation) in addition to KIAA1549-BRAF fusion and synaptophysin expression [3]. In supratentorial compartment, dysembryoplastic neuroepithelial tumors or gangliogliomas represent the most frequent differential diagnoses. Dysembryoplastic neuroepithelial tumors usually exhibit FGFRI alteration (point mutations and internal tandem duplication) and lack of KIAA1549-BRAF fusion whereas gangliogliomas demonstrate frequent $B R A F V 600 E$ mutation in addition to CD34 expression [8, 9]. However, KIAA1549-BRAF fusion has also been recorded in gangliogliomas as well as alteration in the FGFRl gene can be observed in pilocytic astrocytomas. Finally, in small biopsies, when Rosenthal fibers are lacking, and if an infiltrative pattern is observed, differential diagnosis with diffuse glioma might be questionable especially in cases occurring in the midline. In these cases, when $H 3 K 27$ histone mutation is lacking, evidence for KIAA1549-BRAF fusion is of utmost importance to assess pilocytic astrocytoma diagnosis. In our study, KIAA1549-BRAF fusion was recorded in 20/40 pilocytic astrocytomas (50\%), 3/18 gangliogliomas (16.7\%), and in 4/27 (14.8\%) of neuroepithelial tumors that remained difficult to classify (one pilocytic astrocytoma versus ganglioglioma and three pilocytic astrocytomas versus diffuse gliomas), but in none of the dysembryoplastic neuroepithelial tumors. Whether true gangliogliomas display KIAA1549-BRAF fusion or whether these tumors represent misclassified pilocytic astrocytomas is still a matter of debate. Nevertheless, because KIAA1549-BRAF fusion constitutively activates the MAP kinase pathway, this alteration represents a target for drugs such as MEK inhibitors, and therefore, the detection of this genetic abnormality is of utmost importance in these tumors in the context of clinical trials. Indeed, searching for KIAA1549$B R A F$ fusion will be done in all samples from patients that

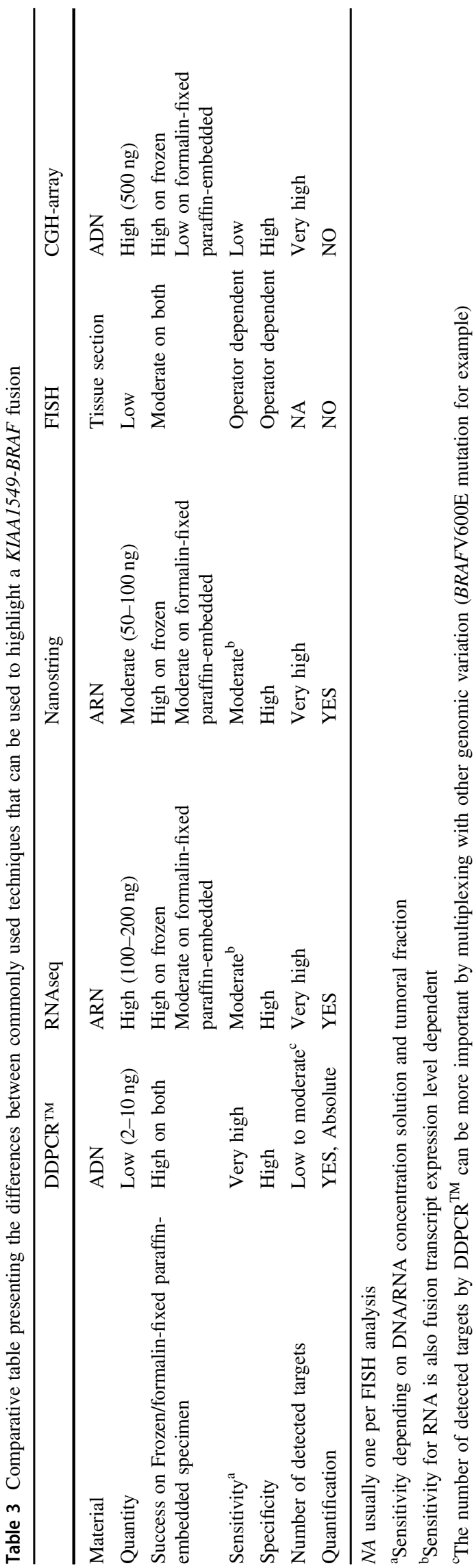


will be included in the incoming SIOP-LOGGIC phase III clinical trial. In the context of KIAA1549-BRAF fusion gene, the most reliable KIAA1549 exon to target to detect KIAA1549-BRAF fusion was exon 4. Importantly, we also showed that appropriate combinations of primers might be used to define which exon (15 or 16) of the KIAA1549 gene is involved in the KIAA1549-BRAF fusion. However, the correlation with RNAseq results was not $100 \%$ and in routine practice it is not currently necessary to decipher the different combinations of KIAA1549-BRAF fusion transcripts. We also learnt from our study that the highest mean copy number variation value of the ratio between two exons analyzed was never above 3 , as expected for a duplication involving one allele in almost all cells.

To conclude, the present study showed the usefulness of DDPCR $^{\mathrm{TM}}$ to assess KIAA1549-BRAF fusion from DNA extracted from formalin-fixed paraffin-embedded tissue. Evidence for $B R A F$ duplication is both necessary and sufficient to predict KIAA1549-BRAF fusion.

Acknowledgements This work was supported by grants from Institut National du Cancer (grant INCa-DGOS-Inserm 6038) and by grants from the A Kids' Brain Tumor Cure/PLGA (Pediatric Low-Grade Astrocytoma) foundation (PLGAO CR3097J7) under the European SIOP-LGG preclinical trial consortium. We thank the Cancéropôle PACA (2017-01), the GEFLUC Marseille Provence (Groupement Entreprises Françaises Lutte contre le Cancer - CT3097S5GEFL0), the ARTC-Sud patients's association (Association pour le Recherche sur les Tumeurs Cérébrales), and the ACCL association (Association Cassandra contre la leucémie et les cancers pédiatriques). We also thank the APHM Tumor Bank (authorization number: AC2013-1786; CRB BB-0033-00097) for retrieving samples.

\section{Compliance with ethical standards}

Conflict of interest The authors declare that they have no conflict of interest.

\section{References}

1. Ohgaki H, Kleihues P. Population-based studies on incidence, survival rates, and genetic alterations in astrocytic and oligodendroglial gliomas. J Neuropathol Exp Neurol. 2005;64:479-89.

2. Louis DN, Perry A, Reifenberger G, et al. The 2016 world health organization classification of tumors of the central nervous system: a summary. Acta Neuropathol. 2016;131:803-20.
3. Louis DN, Ohgaki H, Wiestler OD, et al. World Health Organization Classification of Tumours of the Central Nervous System. In: Louis DN, Wiestler OD, Cavenee WK, editors. 4th ed. revised. Lyon: International Agency for Research on Cancer (IARC); 2016. pp. 80-9.

4. Jones DT, Kocialkowski S, Liu L, et al. Tandem duplication producing a novel oncogenic BRAF fusion gene defines the majority of pilocytic astrocytomas. Cancer Res. 2008;68:8673-7.

5. Tatevossian RG, Lawson AR, Forshew T, et al. MAPK pathway activation and the origins of pediatric low-grade astrocytomas. $\mathrm{J}$ Cell Physiol. 2010;222:509-14.

6. Ryall S, Arnoldo A, Krishnatry R, et al. Multiplex detection of pediatric low-grade glioma signature fusion transcripts and duplications using the NanoString nCounter system. J Neuropathol Exp Neurol. 2017;76:562-70.

7. Belgrader P, Tanner SC, Regan JF, et al. Droplet digital PCR measurement of HER2 copy number alteration in formalin-fixed paraffin-embedded breast carcinoma tissue. Clin Chem. 2013;59:991-4.

8. Fina F, Barets D, Colin C, et al. Droplet digital PCR is a powerful technique to demonstrate frequent FGFR1 duplication in dysembryoplastic neuroepithelial tumors. Oncotarget. 2017;8:2104-13.

9. Chappe C, Padovani L, Scavarda D, et al. Dysembryoplastic neuroepithelial tumors share with pleomorphic xanthoastrocytomas and gangliogliomas BRAF(V600E) mutation and expression. Brain Pathol. 2013;23:574-83.

10. Jones DT, Hutter B, Jager N, et al. Recurrent somatic alterations of FGFR1 and NTRK2 in pilocytic astrocytoma. Nat Genet. 2013;45:927-32.

11. Tian Y, Rich BE, Vena N, et al. Detection of KIAA1549-BRAF fusion transcripts in formalin-fixed paraffin-embedded pediatric low-grade gliomas. J Mol Diagn. 2011;13:669-77.

12. Zhang J, Wu G, Miller $\mathrm{CP}$, et al. Whole-genome sequencing identifies genetic alterations in pediatric low-grade gliomas. Nat Genet. 2013;45:602-12.

13. Tomic TT, Olausson J, Wilzen A, et al. A new GTF2I-BRAF fusion mediating MAPK pathway activation in pilocytic astrocytoma. PLoS ONE. 2017;12:e0175638.

14. Cin H, Meyer C, Herr R, et al. Oncogenic FAM131B-BRAF fusion resulting from $7 \mathrm{q} 34$ deletion comprises an alternative mechanism of MAPK pathway activation in pilocytic astrocytoma. Acta Neuropathol. 2011;121:763-74.

15. Gupta K, Orisme W, Harreld JH, et al. Posterior fossa and spinal gangliogliomas form two distinct clinicopathologic and molecular subgroups. Acta Neuropathol Commun. 2014;2:18.

16. Badiali M, Gleize V, Paris S, et al. KIAA1549-BRAF fusions and IDH mutations can coexist in diffuse gliomas of adults. Brain Pathol. 2012;22:841-7.

17. Rodriguez FJ, Schniederjan MJ, Nicolaides T, et al. High rate of concurrent BRAF-KIAA1549 gene fusion and $1 \mathrm{p}$ deletion in disseminated oligodendroglioma-like leptomeningeal neoplasms (DOLN). Acta Neuropathol. 2015;129:609-10.

\section{Affiliations}

\section{Romain Appay ${ }^{1,2}$. Frédéric Fina ${ }^{1,3} \cdot$ Nicolas Macagno ${ }^{1} \cdot$ Laëtitia Padovani $^{4}$ - Carole Colin ${ }^{2}$ - Doriane Barets ${ }^{1}$. Joanna Ordioni ${ }^{1}$ - Didier Scavarda ${ }^{5}$. Felice Giangaspero ${ }^{6,7}$ - Manuela Badiali ${ }^{8} \cdot$ Andrey Korshunov $^{9}$. Stefan M. Pfister ${ }^{10,11,12} \cdot$ David T.W. Jones $^{10,12}$ - Dominique Figarella-Branger ${ }^{1,2}$}

1 APHM, Hôpital de la Timone, Service d'Anatomie Pathologique et de Neuropathologie, Marseille, France
2 Aix-Marseille Univ, CNRS, INP, Inst Neurophysiopathol, Marseille, France 
3 APHM, Unité Développement Technique, Marseille, France

4 APHM, Hôpital de la Timone, Service de Radiothérapie, Marseille, France

5 APHM, Hôpital de la Timone, Service de Neurochirurgie Pédiatrique, Marseille, France

6 Department of Radiological, Oncological and Pathological Science, Sapienza University, Rome, Italy

7 Neuromed Institute, IRCCS, Isernia, Pozzilli, Italy

8 Laboratory of Genetics and Genomics, Microcitemico Pediatric Hospital, Cagliari, Italy
9 CCU Neuropathology, German Cancer Research Center and Department of Neuropathology, Heidelberg University, Heidelberg, Germany

10 Hopp Children's Cancer Center at the NCT Heidelberg (KiTZ), Heidelberg, Germany

11 Department of Pediatric Oncology, Hematology, Immunology and Pulmonology, Heidelberg University Hospital,

Heidelberg, Germany

12 Division of Pediatric Neurooncology, German Cancer Consortium (DKTK), German Cancer Research Center (DKFZ),

Heidelberg, Germany 